\title{
Measurement of High-Molecular-Weight Polycyclic Aromatic Hydrocarbons in Soils by Particle Beam High-Performance Liquid Chromatography-Mass Spectrometry
}

\author{
Christopher M. Pace \\ Lockheed Environmental Systems and Technologies, Las Vegas, Nevada, USA
}

\section{Leon D. Betowski}

U.S. Environmental Protection Agency, Environmental Monitoring Systems Laboratory, Las Vegas, Nevada, USA

\begin{abstract}
Polycyclic aromatic hydrocarbons (PAHs) comprise a class of potentially hazardous compounds of concern to the U.S. EPA. The application of particle-beam (PB) liquid chromatography-mass spectrometry (LC-MS) to the measurement of high-molecular-weight PAHs was investigated. Instrument performance was evaluated for $16 \mathrm{PAHs}$ in the molecular weight range 300-450 u. The PAHs were separated by reverse-phase high-performance liquid chromatography via a polymeric octadecylsilica (C-18) packing and gradient elution with methanol-tetrahydrofuran. On-column instrument detection limits, as measured by selected ion monitoring on the singly charged molecular ion of each $\mathrm{PAH}$, were found to be $0.15-0.60$ ng for PAHs with molecular weights up to $352 \mathrm{u}$ and 2-4 ng for PAHs with molecular weights greater than $352 \mathrm{u}$. Instrument response was generally linear for PAHs with molecular weights 300-352 u and generally nonlinear for PAHs with molecular weights greater than $352 \mathrm{u}$. The PB electron impact mass spectra of the PAHs were found to vary with the ion distribution ratio of the singly charged molecular ion to the doubly charged molecular ion, dependent on molecular weight, ion source temperature, and concentration. Analysis by PB LC-MS was applied to extracts of PAH-spiked soil and a PAH-contaminated soil from the Pacific Northwest. Target analyte concentrations in the PAH-contaminated soil ranged from 0.85 to $84 \mu \mathrm{g} / \mathrm{g}$. Quantitative estimates for nontarget PAHs also were determined. Analysis of a second soil extract from a hazardous waste site in the northeast part of the United States displayed isomeric patterns of high-molecular-weight PAHs similar to those of the Pacific Northwest extract. (J Am Soc Mass Spectrom 1995, 6, 597-607)
\end{abstract}

$\mathrm{P}$ olycyclic aromatic hydrocarbons (PAHs) comprise a class of hazardous compounds that are widely distributed throughout the environment as complex mixtures and are of interest to the U.S. EPA. Certain PAH isomers are known potent mutagens or carcinogens [1]. Most studies of PAHs in environmental samples have focused on PAHs that contain six fused rings or fewer (MW $<300 \mathrm{u}$ ), even though the presence of high-molecular-weight PAHs (MW > $300 \mathrm{u}$ ) has been demonstrated in many environmental samples contaminated with carbon black, coal tar, petroleum, diesel fuel, and associated by-products. Evidence suggests that high-molecular-weight PAHs are

Address reprint requests to Leon D. Betowski, U.S. EPA, National Exposure Research Laboratory, Characterization Research Division, P.O. Box 93478, Las Vegas, NV 89193-3478. the third most abundant molecular species in the interstellar regions of the universe [2]. Toxicity and analytical characterization data on high-molecular-weight PAHs are limited because purified materials are scarce. Several dibenzopyrenes (MW $302 \mathrm{u}$ ) are highly carcinogenic [3], and dibenzo( $c d, l m)$ perylene (MW $326 \mathrm{u})$ is a moderate carcinogen [4]. Grimmer et al. [5], who interpreted mouse skin studies, attributed about 50\% of the total carcinogenic activity of an extract from a coal combustion effluent to the high-molecular-weight PAH fraction. Consequently, the Environmental Monitoring Systems Laboratory-Las Vegas (EMSL-LV) instituted the present study to evaluate particle beam (PB) liquid chromatography-mass spectrometry (LCMS) to measure a limited set of targeted high-molecular-weight PAHs in a complex environmental sample.

Some of the problems associated with the determination of high-molecular-weight PAHs in environmen- 
tal samples include low vapor pressure, solubility decline with increasing molecular weight, rapid increase in the number of isomeric possibilities with increasing molecular weight, inability of routinely acquired mass spectra to distinguish isomers, and lack of readily available analytical reference standards. Despite difficulties, a considerable amount of data concerning high-molecular-weight PAHs have been accumulated in recent years. Many of the analytical techniques employed to study high-molecular-weight PAHs make use of high-performance liquid chromatography (HPLC) to isolate sample components followed by ancillary measurement by ultraviolet/visible (UV/vis) spectrophotometry, fluorescence spectroscopy, or mass spectrometry.

Schmidt et al. [6] used thin layer chromatography and HPLC to isolate 18 PAHs with molecular weights 300 and $302 \mathrm{u}$ from hard-coal flue gas condensate. Eleven PAHs were unambiguously identified by gas chromatography, mass spectrometry, UV, and fluorescence detection. Peaden et al. [7] utilized HPLC with mass spectrometry and fluorimetry to identify tentatively more than 40 PAHs in the molecular weight range 300-448 $u$ in a carbon black extract. Hirata et al. [8] subsequently compared capillary gas chromatography (GC) with capillary HPLC for the analysis of a carbon black and a coal-tar extract. Although only a few PAHs were unambiguously identified, PAHs of up to eight fused rings were observed by gas chromatography-mass spectrometry (GC-MS) on a short, thermally stabilized glass capillary, and PAHs of up to 11 fused rings could be inferred by capillary HPLC. Hirose et al. [9] used a packed capillary liquid chromatography (LC) column in conjunction with mass spectrometry to identify tentatively over 100 PAHs with molecular weights up to $400 \mathrm{u}$ in a carbon black extract. Colmsjo and Ostman [10] used HPLC to isolate PAHs with molecular weights up to $400 \mathrm{u}$, as determined by off-line mass spectrometry, from a carbon black extract; nine of these were unambiguously identified by Shpol'skii fluorescence. Similarly, Wise et al. [11] identified $13 \mathrm{PAH}$ isomers with molecular weight $302 u$ in a coal tar extract by HPLC with off-line mass spectrometry in conjunction with Shpol'skii fluorescence.

To avoid the time-consuming and tedious fraction collection and off-line measurements, some investigators have examined on-line measurement of highmolecular-weight PAHs. Romanowski et al. [12] determined PAHs with molecular weights up to $456 \mathrm{u}$ in a coal tar extract by using GC-MS with a modified high temperature interface. Fetzer et al. [13] used conventional HPLC with photodiode array detection to identify 13 high-molecular-weight PAHs in diesel exhaust particulates; identification was based on comparison of $\mathrm{UV} / \mathrm{vis}$ spectra and retention times with those of authentic standards synthesized in their laboratory. Wright et al. [14] utilized supercritical fluid chromatography with a capillary column and pentane as the mobile phase to demonstrate elution of PAHs that contained up to an estimated 12 fused rings from a carbon black extract; detection was by on-line fluorescence, but the feasibility of detection by mass spectrometry by using an atmospheric pressure ionization source was discussed. More recently, Wise et al. [15] measured several PAHs with molecular weights 278 and $302 \mathrm{u}$ in four environmental standard reference materials. Normal-phase LC was used to isolate the $\mathrm{PAH}$ isomer groups, which were then analyzed by reversed-phase $L C$ with fluorescence detection. Doerge et al. [16] developed PB LC-MS methods to detect and measure PAHs and oxygenated metabolites in sediment and water samples from the Exxon Valdez oil spill in Alaska. Molecular weights of analytes ranged from 228 to $308 \mathrm{u}$. Perreault et al. [17] demonstrated the use of a moving belt interface to couple conventional HPLC with a mass spectrometer in an effort to characterize high-molecular-weight PAHs in an extract of a coal-tar pond sample; many PAHs in the molecular weight range 326-580 u were observed.

In a previous study sponsored by EMSL-LV, a PB LC-MS-based method was developed to measure the levels of 12 PAHs with molecular weights that ranged from 228 to $302 \mathrm{u}$ in soils and sediments [18]. One result of that work was evidence for the presence of high-molecular-weight PAHs (MW > $300 \mathrm{u}$ ) in an environmental soil sample. The work described in our current report is an examination of PB LC-MS analysis for the measurement of high-molecular-weight PAHs. Instrument response characteristics, solubility, chromatographic conditions, sample preparation, and analysis of environmental samples were all investigated.

\section{Experimental}

\section{Reagents}

HPLC grade chlorobenzene, methanol, and tetrahydrofuran were obtained from Burdick and Jackson (B \& J; Muskegon, MI). HPLC grade tetrahydrofuran (THF) also was obtained from J.T. Baker (Phillipsburg, NJ). Coronene and diindeno $\left(1,2,3-c d: 1^{\prime}, 2^{\prime}, 3^{\prime}-l m\right)$ perylene were obtained from Aldrich Chemical Co. (Milwaukee, WI). Decacyclene was obtained from Chem Service, Inc. (West Chester, PA). Dibenzo $(a, e)$ pyrene, dibenzo $(a, h)$ pyrene, and dibenzo( $a, i)$ pyrene were obtained from Chemsyn Science Laboratories (Lenexa, KS). Benzo(a)coronene, naphtho(2,3-b)fluoranthene, naphtho(1,2,3,4-ghi)perylene, and naphtho(2,3-e) pyrene were obtained from Promochem $\mathrm{GmbH}$ (Wesel, Federal Republic of Germany). Benzo(a)naphtho(8,1,2$c d e)$ naphthacene, dibenzo $(a, j)$ coronene, dibenzo( $h, r s t)$ pentaphene, dibenzo $(c d, l m)$ perylene, naphtho(2,3-a)coronene, and pyranthene were obtained from Chevron Research Co. (Richmond, CA). The PAH reference compounds were evaluated for purity. Coronene and the three dibenzopyrenes were marketed as > $90 \%$ purity. The remainder of the reference PAHs were 
evaluated for purity by HPLC with UV/vis, fluorescence, and PB mass spectrometry detection. Only three reference PAHs were found to be less than $90 \%$ pure: dibenzo( $a, j)$ coronene $(72 \%)$, dibenzo $(c d, I m)$ perylene (82\%), and pyranthene (78\%). Stock solution concentrations of these three PAHs were corrected based on their purities. Stock solution concentrations of those targeted PAHs estimated as better than $90 \%$ pure were not corrected.

\section{Instrumentation}

The LC-MS system consisted of a Hewlett-Packard (HP; Avondale, PA) 1090L liquid chromatography, HP 59980A PB interface, and an HP 5988A quadrupole mass spectrometer. Instrument operation and data processing were under the control of an 59970 mass spectrometry Chemstation.

The liquid chromatograph used a $250-\mathrm{mm} \times 4.6-\mathrm{mm}$ i.d. Vydac 201 TP polymeric C-18 column with $5-\mu \mathrm{m}$ particle size to separate the targeted PAHs (Separations Group, Hesperia, CA). The HPLC was operated at a flow rate of $0.4 \mathrm{~mL} / \mathrm{min}$ and a column compartment temperature of $40{ }^{\circ} \mathrm{C}$. The nonaqueous reverse-phase solvent program consisted of isocratic operation for the first $8 \mathrm{~min}$ with $100 \%$ methanol, programming to $60: 40$ methanol:THF in $32 \mathrm{~min}$, then to $100 \% \mathrm{THF}$ in $20 \mathrm{~min}$, and isocratic with $100 \% \mathrm{THF}$ for an additional $5 \mathrm{~min}$ (65-min total analysis time). Reequilibration of the column was accomplished by employing the initial conditions for $10 \mathrm{~min}$ prior to the next injection.

The PB interface was operated at a desolvation chamber temperature of $45^{\circ} \mathrm{C}$. The nebulizer capillary position and helium flow rate were set to maximize mass spectrometry response to the $m / z 302$ ion from 10 -ng dibenzo $(a, h)$ pyrene. This was accomplished by using selected ion monitoring (SIM) at $m / z 302$ under flow-injection conditions (bypassing the analytical column) with a mobile phase composition of methanol:THF (1:1) at a flow rate of $0.4 \mathrm{~mL} / \mathrm{min}$.

The mass spectrometer was operated with a source temperature of 325 or $350^{\circ} \mathrm{C}, 70-\mathrm{eV}$ electron energy, and an emission current of $300 \mu \mathrm{A}$, and it was tuned with perfluorotributylamine to maximize the $m / z 414$ ion intensity with a mobile phase composition of methanol:THF (1:1) at a flow rate of $0.4 \mathrm{~mL} / \mathrm{min}$. The mass spectrometer was scanned from 140 to $600 \mathrm{u}$ at a rate of 0.4 scans/s. For SIM data acquisition, a dwell time of $225 \mathrm{~ms}$ was used for each PAH molecular ion, which resulted in 0.4 cycles/s (eight ions monitored simultaneously).

\section{Sample Preparation}

The PAHs were extracted from spiked soil and a PAH-contaminated soil from a carbon electrode disposal area located at the site of a former aluminum
Table 1. PAHs selected for study

\begin{tabular}{|c|c|c|}
\hline No. ${ }^{8}$ & Compound & $\mathrm{MW}^{\mathrm{b}}$ \\
\hline 1 & Coronene & 300 \\
\hline 2 & Dibenzola,e pyrene & 302 \\
\hline 3 & Dibenzola,hlpyrene & 302 \\
\hline 4 & Dibenzola,i)pyrene & 302 \\
\hline 5 & Naphtho(2,3-e)pyrene & 302 \\
\hline 6 & Naphtho(2,3-b)fluoranthene & 302 \\
\hline 7 & Dibenzolcd, /m)perylene & 326 \\
\hline 8 & Naphtho(1,2,3,4-ghi)perylene & 326 \\
\hline 9 & Benzola)coronene & 350 \\
\hline 10 & Benzo(a)naphthol(8,1,2-cde) naphthacene & 352 \\
\hline 11 & Dibenzolh.rst)pentaphene & 352 \\
\hline 12 & Pyranthene & 376 \\
\hline 13 & Dibenzola,jlcoronene & 400 \\
\hline 14 & Diindenol1,2,3-cd:1', $\left.2^{\prime}, 3^{\prime}-/ m\right)$ perylene & 400 \\
\hline 15 & Naphtho(2,3-a)coronene & 400 \\
\hline 16 & Decacyclene & 450 \\
\hline
\end{tabular}

Numbers correspond to Figure 1.

'MW is the molecular weight (units).

plant in the Pacific Northwest. Soil (10 g) was extracted with 300-mL chlorobenzene in a Soxhlet apparatus for $24 \mathrm{~h}$. The resulting extract was then reduced to a volume of $20 \mathrm{~mL}$ on a rotary evaporator. The extract was then evaporated to near dryness in a heated sand bath under a stream of nitrogen. The extract residue was sonicated with two 5-mL portions of methanol:water (80:20). The $5-\mathrm{mL}$ portions were passed through a $6-\mathrm{mL}$ reservoir $\mathrm{C}-18(1000-\mathrm{mg})$ solid phase extraction (SPE) cartridge (B \& J). A total of $10 \mathrm{~mL}$ of chlorobenzene then was used to elute the high-molecular-weight PAHs from the cartridge. The SPE cleanup was found to remove adequately the bulk of lowmolecular-weight PAHs from the extract. The volume of the chlorobenzene extract was reduced to $1 \mathrm{~mL}$ for analysis of the spiked soil samples.

\section{Results and Discussion}

Sixteen PAHs initially were selected for the study. Their names and molecular weights are listed in Table 1 , and their corresponding molecular structures are displayed in Figure 1. The PAHs were selected primarily on the basis of their availability as pure standards.

To gain perspective on PB sensitivity, selected high-molecular-weight $\mathrm{PAHs}$ were injected under flow-injection conditions and the response was measured in the full-scan mode and then by SIM on the molecular ion of interest. The mobile-phase composition was methanol:THF (1:1) at a flow rate of 0.4 $\mathrm{mL} / \mathrm{min}$. An ion-source temperature of $325{ }^{\circ} \mathrm{C}$ was used. These experiments were.conducted by injection of decreasing amounts of analyte until the resulting signal was observed to be about three times the baseline noise. The estimated instrument detection limits 


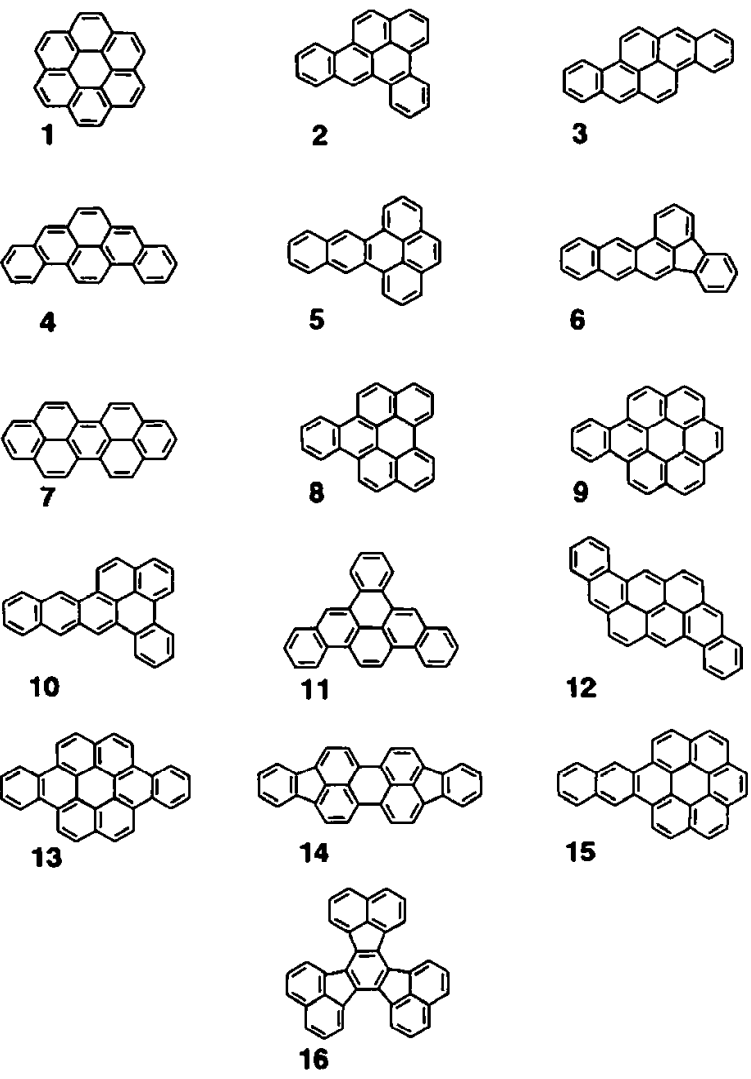

Figure 1. Structures of PAHs selected for study. Names and molecular weights are listed in Table 1.

(IDLs) were determined to range from 10 to $54 \mathrm{ng}$ in the full-scan mode and from 0.1 to $1.3 \mathrm{ng}$ by SIM. Thus, SIM was 1-2 orders of magnitude more sensitive than the full-scan mode.

A transitional region in IDLs was observed between molecular weights 350 and $376 \mathrm{u}$; the IDL increased by a factor of 2 to 3 in the full-scan mode and a factor of $\sim 10$ in SIM. The observed decrease in sensitivity with increasing molecular weight in the full-scan and SIM modes might be attributed to high-mass bias of the quadrupole instrument and perhaps to the effects of ion-source temperature. The operational ion-source temperature limit of $350^{\circ} \mathrm{C}$ may not be high enough to vaporize the higher mass PAHs to the extent (or at the same rate) that lower mass PAHs are vaporized, which resulted in a decrease in the effective ionization efficiency. Additionally, for SIM measurement, the increase in IDLs also may be attributed to the tendency of PB electron impact (EI) of PAHs to yield proportionately more abundant doubly charged ions than singly charged molecular ions at higher molecular weight. Only the singly charged molecular ions were measured in these experiments.

The PB EI full-scan mass spectra of the high-molecular-weight (MW $>300 \mathrm{u}$ ) PAHs are similar to the mass spectra of low-molecular-weight (MW $<300 \mathrm{u})$ PAHs in that they are relatively simple. The two principal ions formed are the molecular ion and the doubly charged molecular ion. Significant intensity also was observed at mass-to-charge ratio values that correspond to the loss of one to four hydrogens from the principal ions. Figure 2 displays the PB EI mass spectra of dibenzo( $a, h)$ pyrene (MW $302 \mathrm{u}$ ) and naphtho(2,3a)coronene (MW $400 \mathrm{u}$ ). The most apparent trend in the PB mass spectra of PAHs generated in this study is the increase in relative abundance of the doubly charged molecular ion with increasing molecular weight. For PAHs with molecular weights greater than approximately $350 \mathrm{u}$, the doubly charged molecular ion accounts for the base peak. This trend might be rationalized by the fact that as the size of the PAH molecule increases, the second ionization potential decreases, which increases the probability that interaction with a $70-\mathrm{eV}$ electron will result in a doubly charged ion. Other experimental data indicate that the PB mass spectra of the high-molecular-weight PAHs are variable and depend not only on molecular weight, but also on ion source temperature and concentration.

During some of the initial PB experiments, it was discovered that instrument response to PAHs varied when different brands of THF were used in the mobile phase. The EI mass spectra of the THF (MW $72 u$ ) that gave an enhanced response displayed a significant ion at $m / z 87(\mathrm{~m} / z 174$ by negative ion chemical ioniza-

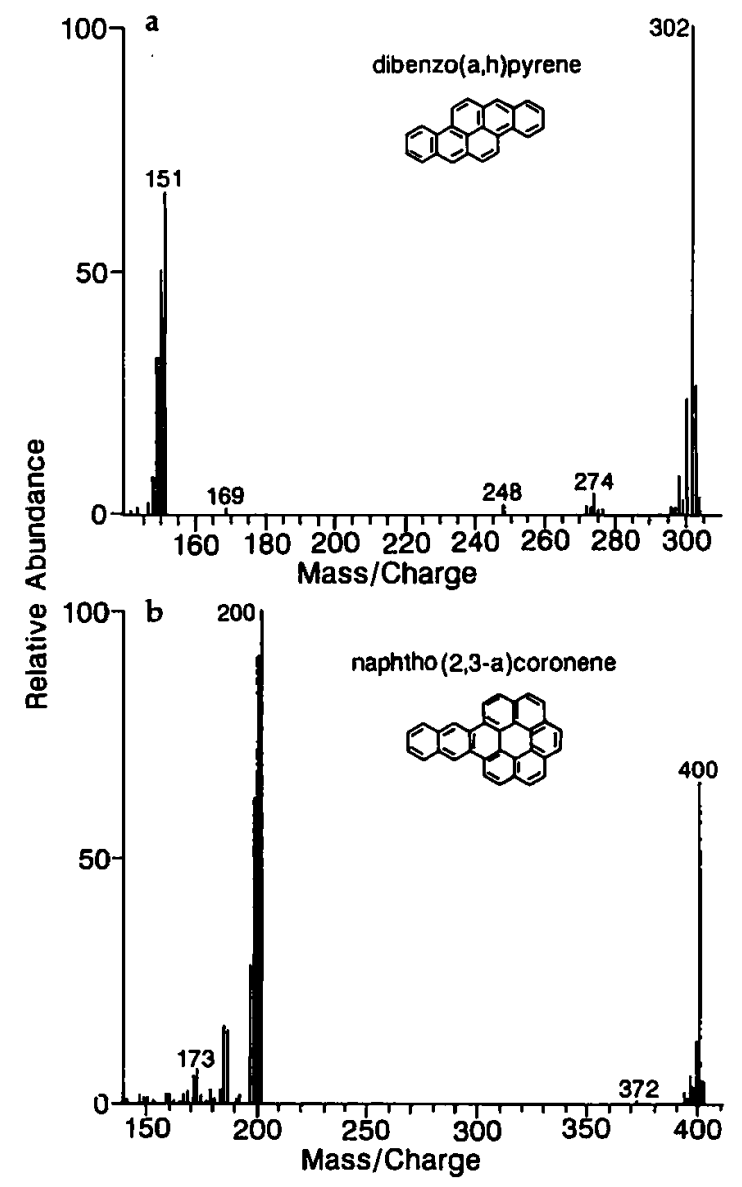

Figure 2. Mass spectra of (a) dibenzo( $a, h$ )pyrene (MW $302 u$ ) and (b) naphtho(2,3-a)coronene (MW $400 \mathrm{u}$ ). 
tion with $\mathrm{CO}_{2}$ ), whereas the THF that did not give an enhanced response did not contain this background ion. THF is known to degrade on exposure to air to form peroxides, and the enhanced response could be due to a "carrier effect" caused by impurities [19].

An experiment was conducted to estimate the relative solubilities of the higher mass PAHs in seven different solvents by using diindenoperylene as the test solute. The following solvation order was observed: THF > chlorobenzene > methylene chloride > benzene $>$ toluene $>$ xylenes $\rightarrow$ isooctane. Because THF has the tendency to form peroxides, chlorobenzene is a better choice for stock solutions, extractions, or for any situation where the PAHs are to be in solution for a prolonged period of time. The diindenoperylene selected for this study may not be representative of all PAH structural types, and the preceding solvation order should be considered in the most general sense.

\section{Chromatography}

A Vydac 201 TP polymeric C-18 reverse-phase column was selected for this work. The literature on the HPLC separation of the high-molecular-weight PAHs reports that polymeric $\mathrm{C}-18$ bonded-phase packings achieve better separation of isomeric PAHs than octadecylbonded silicas [11, 20-22]. Elution behavior of the smaller PAHs (MW < $300 \mathrm{u}$ ) on the polymeric C-18 packings has been correlated to molecular size and shape. The length-to-breadth ratio ( $\mathrm{L} / \mathrm{B})$ is a parameter developed to characterize PAH shape [23]. PAHs that have larger $\mathrm{L} / \mathrm{B}$ ratios are retained more than those with smaller $\mathrm{L} / \mathrm{B}$ ratios. For larger PAHs, the degree of planarity becomes an important factor in determining elution behavior on the polymeric C- 18 bonded phases $[20,21]$. The planar PAHs are retained longer than nonplanar PAHs. Retention anomalies occur when solvent-solute interactions induce nonplanarity in otherwise planar PAHs. These interactions are thought to be a function of intramolecular steric strain in the larger PAH molecules [24].

Most mobile-phase compositions for nonaqueous reverse-phase separations of large PAHs utilize acetonitrile or methanol as the weak solvent and ethyl acetate or methylene chloride as the strong solvent. Chlorobenzene and THF have been shown to be the strongest solvents for elution of high-molecular-weight PAHs, followed by chloroform and methylene chloride [22]. Methanol was selected as the weak solvent and THF was selected as the strong solvent for this work. Both selections were based primarily on PB sensitivity. By direct comparison under flow-injection conditions with dibenzo( $a, h)$ pyrene, methanol was found to be better than acetonitrile for sensitivity and, likewise, THF was better than methylene chloride.

A typical SIM PB LC-MS total ion chromatogram of the targeted PAHs is shown in Figure 3. An ion source

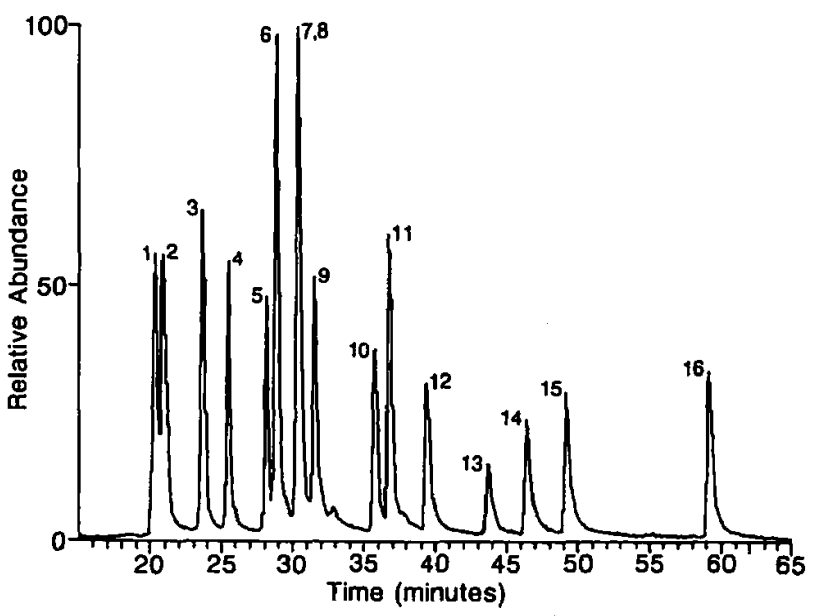

Figure 3. SIM PB LC-MS total ion chromatogram of 16 highmolecular-weight PAH standards. Peak identities are listed in Table 2.

temperature of $325{ }^{\circ} \mathrm{C}$ was used. Peak identities, along with retention times, capacity factors $\left(k^{\prime}\right)$, and retention-time percent relative standard deviations (RSD) are listed in Table 2 . The solvent program was capable of near baseline resolution for most components. Of the two unresolved pairs, one pair-naphtho(2,3$e)$ pyrene and dibenzo(a,e)pyrene-was better than $50 \%$ resolved; the other pair-dibenzo( $a, h)$ pyrene (MW 302 $u)$ and dibenzo( $h, r s t)$ pentaphene (MW $352 \mathrm{u}$ ) - can be mass-resolved.

Column temperature can play a significant role in the elution behavior of the high-molecular-weight PAHs on polymeric C-18 packings. Jinno et al. [25] reported that at higher temperatures the polymeric C-18 selectivity for planarity decreased and was lost at temperatures greater than $40{ }^{\circ} \mathrm{C}$. In previous work sponsored by EMSL-LV, temperature-dependent variation in $\mathrm{PAH}$ retention on the polymeric $\mathrm{C}-18$ packing was reported [18]. Retention times were observed to decrease by as much as $3 \%$ over a 4 -h period as ambient temperature increased. To stabilize retention times, the column temperature must be kept constant. The HP 1090L HPLC used in these studies is equipped with a column oven. However, the oven must be operated at $10^{\circ} \mathrm{C}$ above ambient temperature to achieve precise temperature control. This limits operation to near $40{ }^{\circ} \mathrm{C}$. Under these conditions, percent RSDs were $0.6 \%$ or less (Table 2). Although this may not be the optimum operating temperature, reasonable separation of the targeted PAHs was achieved.

As a practical consideration, injection volume also was investigated. Mismatch between sample solvent and mobile-phase composition can result in distorted peak shapes for early eluting components dependent, in part, on injection volume. Because of solubility considerations, the PAH standards mix was prepared in chlorobenzene, which created a solvent mismatch with the initial mobile-phase condition of $100 \%$ methanol. Under these conditions, $5 \mu \mathrm{L}$ was found to 
Table 2. Targeted PAH chromatographic and response data

\begin{tabular}{|c|c|c|c|c|c|c|c|}
\hline $\begin{array}{l}\text { Peak } \\
\text { no. }\end{array}$ & Compound & MW & RT & $\mathbf{k}^{\prime}$ & $\% R S D(R T)$ & $\%$ RSD (RF) & IDL \\
\hline 1 & Naphtho(2,3-e)pyrene & 302 & 20.40 & 1.91 & 0.5 & 10 & 0.15 \\
\hline 2 & Dibenzol $a, e)$ pyrene & 302 & 20.99 & 2.00 & 0.3 & 7 & 0.15 \\
\hline 3 & Naphtho(2,3-b)fluoranthene & 302 & 23.73 & 2.39 & 0.4 & 7 & 0.15 \\
\hline 4 & Coronene & 300 & 25.58 & 2.65 & 0.3 & 6 & 0.15 \\
\hline 5 & Dibenzola,ilpyrene & 302 & 28.24 & 3.03 & 0.4 & 11 & 0.15 \\
\hline 6 & Naphthol 1,2,3,4-ghilperylene & 326 & 29.00 & 3.14 & 0.4 & 9 & 0.20 \\
\hline 7 & Dibenzola,h)pyrene & 302 & 30.36 & 3.34 & 0.3 & 10 & 0.20 \\
\hline 8 & Dibenzol $h, r s t$ pentaphene & 352 & 30.57 & 3.37 & 0.5 & 26 & 0.50 \\
\hline 9 & Benzola)naphtho(8,1,2-cde) naphthacene & 352 & 31.69 & 3.53 & 0.4 & 10 & 0.45 \\
\hline 10 & Dibenzolcd./m)perylene & 326 & 35.89 & 4.13 & 0.5 & 11 & 0.40 \\
\hline 11 & Benzolalcoronene & 350 & 36.90 & 4.27 & 0.5 & 11 & 0.60 \\
\hline 12 & Decacylene & 450 & 39.56 & 4.65 & 0.3 & 21 & 3.5 \\
\hline 13 & Pyranthene & 376 & 43.81 & 5.26 & 0.5 & 27 & 3.8 \\
\hline 14 & Naphtho(2,3-a)coronene & 400 & 46.59 & 5.66 & 0.6 & 38 & 3.3 \\
\hline 15 & Dibenzola,jlcoronene & 400 & 49.39 & 6.06 & 0.3 & 17 & 2.4 \\
\hline 16 & Diindeno(1,2,3-cd: $1^{\prime}, 2^{\prime}, 3^{\prime}$-Im) perylene & 400 & 59.42 & 7.49 & 0.6 & 32 & 1.8 \\
\hline
\end{tabular}

'Peak no. corresponds to peaks labeled in Figure $3 ; \mathrm{MW}$ is the molecular weight (units); RT is the retention time (minutes); $k$ ' is the capacity factor $\left(V_{0}=2.8 \mathrm{~mL}\right)$; \%RSD (RT) is the retention time percent relative standard deviation ( $\left.n=7\right)$; \%RSD (RF) is the response factor percent relative standard deviation $(n=7$, for most entries); IDL is the instrument detection limit in terms of nanograms.

be the maximum injection volume that did not distort peak shape.

\section{On-Column Instrument Performance}

The on-column PB response to high-molecular-weight PAHs over a range of concentrations was evaluated. The low solubility of the standards with molecular weights greater than $352 \mathrm{u}$ and lack of sufficient fullscan sensitivity required that the analysis be conducted in the SIM operating mode. The targeted PAHs were injected at seven different concentration levels that spanned a concentration range of one hundred fold for most of the targeted PAHs, starting at a low level approximating three times the estimated detection limits to a high concentration 100 times the low level mix. For PAHs with molecular weights 300 and $302 \mathrm{u}$, the concentrations ranged from 0.05 to $5 \mu \mathrm{g} / \mathrm{mL}$, 0.1 to $10 \mu \mathrm{g} / \mathrm{mL}$ for PAHs with molecular weights 326 to $352 \mathrm{u}$, and 0.25 to $25 \mu \mathrm{g} / \mathrm{mL}$ for PAHs with molecular weights 376 to $450 \mathrm{u}$. An ion source temperature of $325{ }^{\circ} \mathrm{C}$ was used. Response factors were calculated as the ratio of integrated signal area to concentration. PB response to 11 of the $16 \mathrm{PAHs}$ exhibited good to excellent linearity (response factor percent RSD < 20\%) over the concentration range and poor linearity for five (Table 2). Poor linearity (response factor percent RSD $>20 \%$ ) was generally observed for those PAHs in the molecular weight range 376 to $450 \mathrm{u}$. Response factors tended to increase with increasing concentration for the majority of the PAHs investigated and was more pronounced for those PAHs with molecular weights greater than $352 \mathrm{u}$. The lack of linearity is not necessarily a problem in terms of quantitative accuracy, pro- vided the response curves are reproducible and can be modeled correctly (e.g., polynomial curve fits or pointto-point calibrations).

The IDLs under the prevailing chromatographic conditions were estimated from the same data set used to establish response curves. In this case, the data that gave a signal intensity approximating three times the signal-to-noise ratio were selected. Table 2 lists the IDLs in terms of nanograms on-column. Again, PB sensitivity for the PAHs in the molecular weight range $376-450 \mathrm{u}$ was less than that in the molecular weight range $300-352 \mathrm{u}$ by a factor of approximately 20 . The sensitivity of on-column analysis is 2-5 times less than by flow injection.

The short-term analytical precision of a mixture that contained 13 high-molecular-weight PAHs was evaluated. An ion source temperature of $350{ }^{\circ} \mathrm{C}$ was used. Each of three standards mixtures at low $(0.12-0.62$ $\mathrm{ng} / \mu \mathrm{L})$, medium (1.5-7.7 $\mathrm{ng} / \mu \mathrm{L}$ ), and high (3.08-15.4 $\mathrm{ng} / \mu \mathrm{L}$ ) concentrations was analyzed in triplicate and the percent RSD for individual analytes at each level was determined. Each single-level triplicate analysis was conducted on the same day. All three levels displayed percent RSD values of $16 \%$ or less. These values indicate acceptable short-term precision for the three levels investigated.

For a rugged method, the initial calibration should be reproducible over an acceptable period of time. The multiday precision was evaluated by analyzing a midlevel standard that contained 13 high-molecularweight PAHs in triplicate over two separate periods of 3 and 4 days. The percent RSDs were all less than $20 \%$, except for diindeno $\left(1,2,3-c d: 1^{\prime}, 2^{\prime}, 3^{\prime}-1 m\right)$ perylene, which had a RSD of $23 \%$ during the 4 -day period. As ex- 
pected, the multiday percent RSDs were higher than those observed on a single day. One explanation for the slightly higher percent RSDs is that the PB probe is physically removed from the vacuum manifold of the mass spectrometer when the instrument is in standby mode (necessary when it is unattended overnight). Small changes in the alignment of the PB interface from day to day could lead to small changes in the instrument response and, hence, higher percent RSDs.

\section{Spiked Soil Recoveries}

Due to low solubility and lack of pure material, three of the original 16 target analytes were left out of the soil spiking experiments because an adequate amount of spiking solution could not be prepared. These three analytes are benzo( $a)$ naphtho( $(8,1,2-c d e)$ naphthacene, dibenzo $(a, j)$ coronene, and naphtho(2,3-a)coronene.

A source of possible interference is the large number and high concentration of low-molecular-weight PAHs (MW $<300 \mathrm{u}$ ) present in samples that contain high-molecular-weight PAHs. In previous PB LC-MS analysis done on PAH-contaminated soils, the lowmolecular-weight PAHs were present at concentrations up to several orders of magnitude greater than individual high-molecular-weight PAHs. A problem caused by the lower mass PAHs is ion-source contamination. High background levels can result when large quantities of organic molecules reach the PB ion source [26]. This may arise because the PB LC-MS mechanism for ionization requires analyte particles to volatilize upon contact with the hot ion-source surface. The volatilization is not instantaneous and is characterized by peak tailing (not observed in the UV/vis chromatogram). The SPE cleanup described in the Experimental section was found to adequately remove the bulk of the low-molecular-weight PAHs without an adverse effect on the recoveries of the target analytes.

Recoveries (average of three) from spiked blank soil $(0.12-0.62 \mu \mathrm{g} / \mathrm{g})$ that used the scheme outlined in the Experimental section ranged from 71 to $95 \%$ for 12 of the 13 analytes examined. One of the analytes (pyranthene) displayed a recovery of only $10 \%$. The recovery percent RSDs ranged from 5.3 to $10.0 \%$ for the 13 analytes examined. The low recovery for pyranthene was determined to be the result of the Soxhlet extraction and not due to the SPE cleanup.

\section{Analysis of a PAH-Contaminated Soil}

Table 3 lists the quantities of the target analytes in the soil. Although mass-spectral quantitation ions and retention times were used in the identification process, the overwhelming number of isomeric possibilities that arise as PAH ring size increases makes positive identification very difficult to obtain. Interference from a much larger closely eluting isomer made accurate
Table 3. PAH results of soil extract ${ }^{\mathrm{a}}$

\begin{tabular}{lccc}
\hline Compound & MW & RT & Conc. \\
\hline \hline Naphtho(2,3-e)pyrene & 302 & 20.90 & 43 \\
Dibenzola,e)pyrene & 302 & 21.57 & 84 \\
Naphtho(2,3-b)fluoranthene & 302 & 24.83 & Int. \\
Coronene & 300 & 26.78 & 42 \\
Dibenzola,i)pyrene & 302 & 29.90 & 10 \\
Naphthol(1,2,3,4-ghi)perylene & 326 & 30.61 & 46 \\
Dibenzola,h)pyrene & 302 & 32.20 & 2.0 \\
Dibenzol $h, r s t)$ pentaphene & 352 & 32.36 & 3.7 \\
Dibenzolcd,Im)perylene & 326 & 37.98 & 9.4 \\
Benzola)coronene & 350 & 38.93 & 21 \\
Decacyclene & 450 & 41.68 & 0,85 \\
Pyranthene & 376 & 46.14 & 18 \\
Diindenol1,2,3-cd:1',2',3'-lm/perylene & 400 & 60.68 & 0.89 \\
\hline
\end{tabular}

aW is the molecular weight (units); RT is the retention time (minutes); conc. is the concentration of analytes $(\mu \mathrm{g} / \mathrm{g})$ in extract; Int. is the interfering unknown MW 302 isomer.

measurement of naphtho(2,3-b)fluoranthene impossible. Figure 4 displays the SIM chromatograms of four of the eight ions $(\mathrm{m} / \mathrm{z} 302,326,352$, and 400$)$ monitored in the analysis of the contaminated soil. The increasing number of potential isomers with increasing
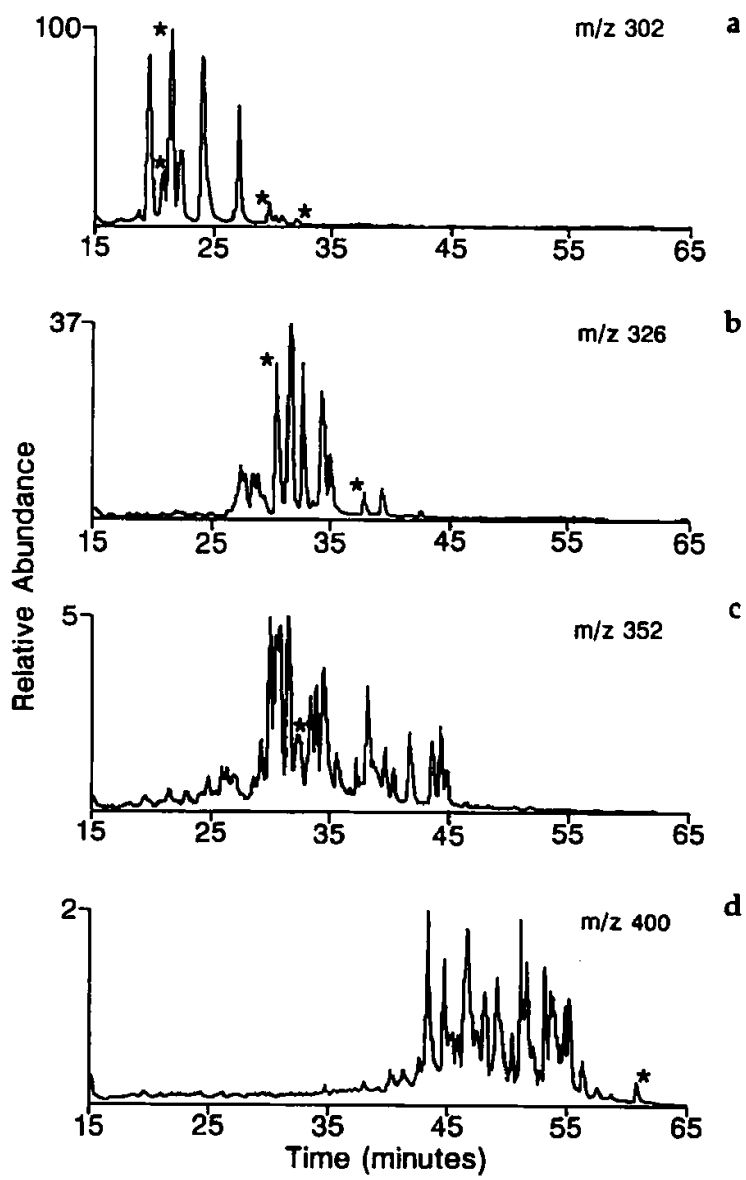

Figure 4. SIM ion chromatograms of (a) $m / z$ 302, (b) $m / z$ 326, (c) $m / z$ 352, and (d) $m / z 400$ from the Pacific Northwest PAHcontaminated soil extract. Targeted PAHs are labled with asterisks. The $y$-axis scalings are relative to the $m / z 302$ abundance. 
molecular weight of PAHs is clearly evident in these chromatograms.

To measure the tentatively identified compounds in the extract, a mixture of three analytes was prepared at two levels that differed by a factor of 10 (roughly encompassing the expected concentration range in the sample) and analyzed under full-scan conditions. The response factors were averaged for each analyte in the two levels and used to measure the nontarget components in the sample. The integrated peak area of the molecular ion was used for the response factor calculation. A response ratio of 1:1 between the standard $\mathrm{PAH}$ analytes and the PAHs measured in the sample is assumed. The three PAHs were selected based on molecular weight, retention time, and stock standard solution concentration. These analytes were used to measure nontarget PAHs in a specific molecular weight range. Naphtho(2,3-b)fluoranthene (MW 302 u) was used to measure PAHs with molecular weights between 284 and $318 \mathrm{u}$; benzo( $a$ )coronene (MW $350 \mathrm{u}$ ) for those between 326 and $356 u$; decacyclene (MW $450 u$ ) for those between 374 and $424 u$.

Figure 5 displays the full-scan PB EI LC-MS chromatogram of the soil extract. The molecular ions for several of the components in this sample are labeled in this figure. The PB full-scan background when scanning above $m / z 140$ is negligible when compared to the apparent baseline in Figure 5. On close inspection the "baseline" actually comprises a large number of closely eluting (and coeluting) compounds. Table 4 presents the quantitative results for the tentatively

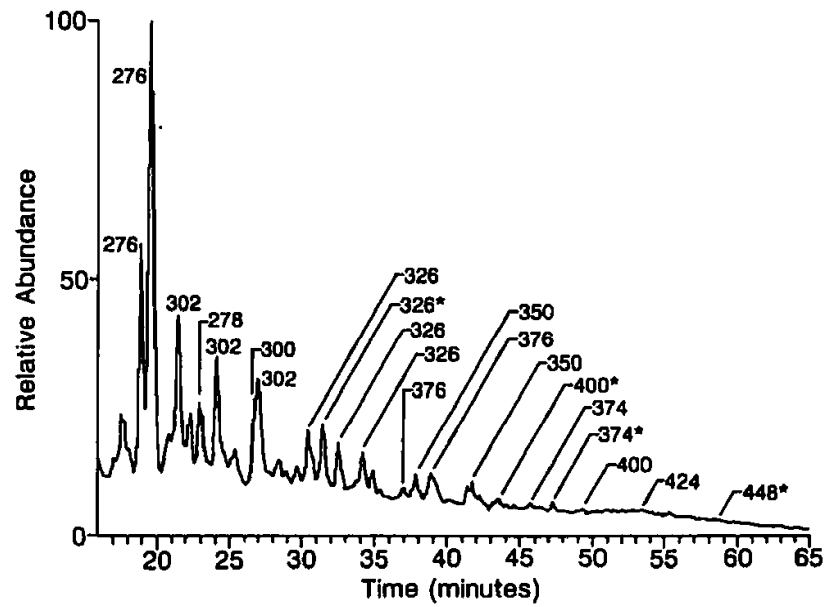

Figure 5. Full-scan PB LC-MS chromatogram of the Pacific Northwest PAH-contaminated soil extract. Molecular ions of several representative PAHs are shown. The mass spectra of those components with asterisks are shown in Figure 6.

identified compounds in the soil extract. Most of these compounds were clustered in groups of isomers with very similar EI mass spectra. This table lists the massto-charge ratio of the molecular ion, the retention time window in which the isomers were observed, and the estimated concentration range of the isomers at each molecular weight. Also listed in this table are the probable molecular formula and a non-isomer-specific identification with the number of isomers observed in parentheses. These identifications were based on the

Table 4. Tentatively identified polycyclic aromatic hydrocarbons in the soil extract ${ }^{\mathrm{a}}$

\begin{tabular}{|c|c|c|c|c|}
\hline$m / z$ & RT range & Formula & $\begin{array}{c}\text { Identification } \\
\text { (number of isomers) }\end{array}$ & $\begin{array}{c}\text { Estimated conc. } \\
\text { range }(\mu g / g)\end{array}$ \\
\hline 284 & $19.4-25.2$ & $\mathrm{C}_{20} \mathrm{H}_{12} \mathrm{~S}$ & Dinaphthothiophene (3) & $6.4-22.6$ \\
\hline 290 & $18.1-25.6$ & Unknown & Unknown $(7)$ & $2.0-25$ \\
\hline 292 & $17.8-30.6$ & $\mathrm{C}_{23} \mathrm{H}_{16}$ & Methyl-278 (9) & $3.3-21$ \\
\hline 300 & $25.4-26.6$ & $\mathrm{C}_{24} \mathrm{H}_{12}$ & Coronene (2) & $12-68$ \\
\hline 302 & $17.2-31.9$ & $\mathrm{C}_{24} \mathrm{H}_{14}$ & Dibenzopyrene/fluoranthene (12) & $2.0-100$ \\
\hline 308 & $19.4-33.3$ & Unknown & Unknown (14) & $1.1-8.7$ \\
\hline 314 & 30.9 & Unknown & Unknown $\{1\rangle$ & 2.0 \\
\hline 316 & $21.4-41.9$ & $\mathrm{C}_{25} \mathrm{H}_{16}$ & Methyl-302 (15) & $0.66-3.1$ \\
\hline 318 & $21.4-37.0$ & Unknown & Unknown (12) & $0.82-1.8$ \\
\hline 326 & $27.3-39.1$ & $\mathrm{C}_{26} \mathrm{H}_{14}$ & Dibenzoperylene (12) & $9.9-64$ \\
\hline 328 & $21.9-42.2$ & $\mathrm{C}_{26} \mathrm{H}_{16}$ & Pyrenylnaphthalene (9) & $2.3-22$ \\
\hline 332 & $26.8-38.6$ & Unknown & Unknown (6) & $1.6-5.3$ \\
\hline 334 & $35.4-44.3$ & Unknown & Unknown (3) & $2.0-2.8$ \\
\hline 350 & $37.1-46.1$ & $\mathrm{C}_{28} \mathrm{H}_{14}$ & Benzocoronene (6) & $3.1-36$ \\
\hline 352 & $29.0-44.6$ & $\mathrm{C}_{28} \mathrm{H}_{16}$ & Dibenzoperylene (18) & $1.6-9.7$ \\
\hline 356 & $37.9-43.5$ & $\mathrm{C}_{26} \mathrm{H}_{12} \mathrm{~S}$ & Benzopyrenodibenzothiophene (4) & $1.2-1.8$ \\
\hline 374 & $45.7-47.2$ & $\mathrm{C}_{30} \mathrm{H}_{14}$ & Naphthocoronene (2) & $15-26$ \\
\hline 376 & $36.9-56.0$ & $\mathrm{C}_{30} \mathrm{H}_{16}$ & Tribenzoperylene (18) & $1.3-22$ \\
\hline 400 & $43.1-60.6$ & $\mathrm{C}_{32} \mathrm{H}_{16}$ & Naphthocoronene (17) & $0.50-2.5$ \\
\hline 424 & $49.8-59.8$ & $\mathrm{C}_{34} \mathrm{H}_{16}$ & Benzonaphthocoronene (13) & $1.5-2.5$ \\
\hline
\end{tabular}

- $m / z$ is the mass-to-charge ratio of molecular ion; $R T$ is the retention time (minutes). 

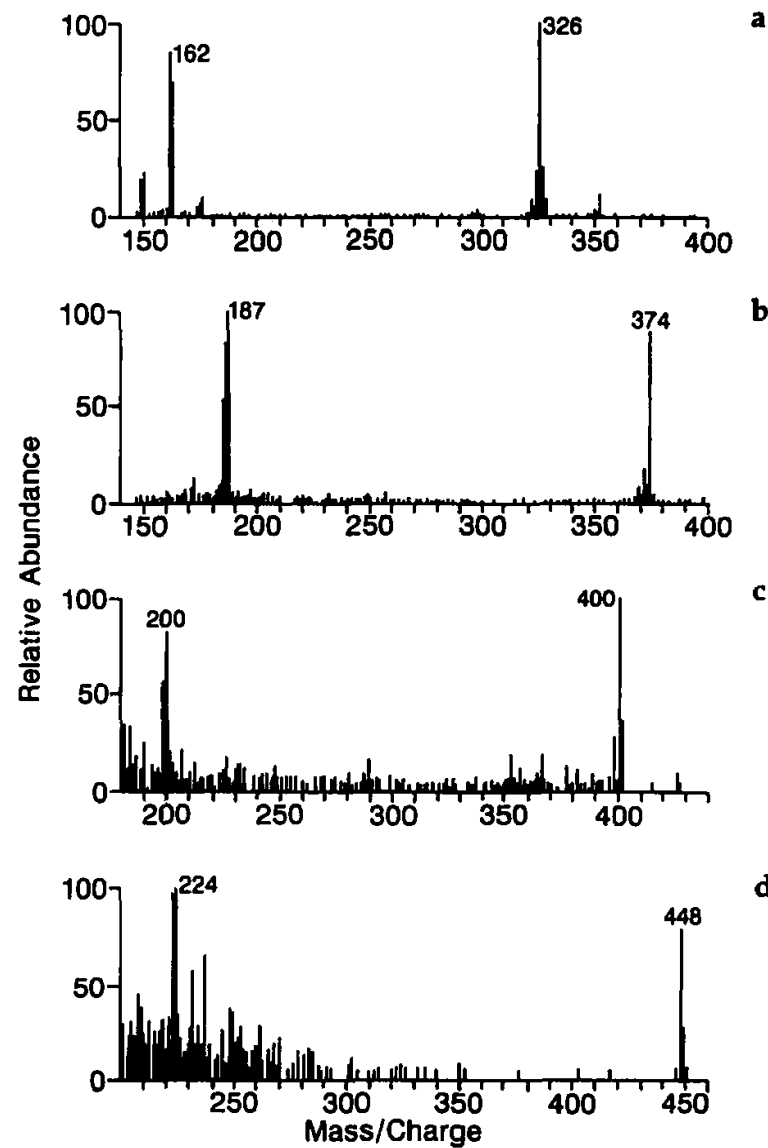

Figure 6. Representative background-subtracted mass spectra obtained from the PB LC-MS analysis of the Pacific Northwest PAH-contaminated soil extract.

mass spectra and information found in the literature. The determination of the number of isomers in Table 4 was accomplished by using ion chromatograms to assess the number of individual peaks and did not take into account coeluting or very low abundance isomers. Figure 6 presents representative examples of the PB LC-MS full-scan background subtracted mass spectra of four PAHs observed in the soil extract.

For comparison, the concentrations of eight commonly measured PAHs that range in molecular weights from 228 to $278 \mathrm{u}$ also were determined by PB LC-MS. PAHs with molecular weights less than $228 \mathrm{u}$ do not efficiently pass through the particle beam interface [18]. The concentrations determined ranged from 71 $\mu \mathrm{g} / \mathrm{g}$ for dibenzo $(a, h)$ anthracene (MW $278 \mathrm{u}$ ) to 2200 $\mu \mathrm{g} / \mathrm{g}$ for benzo(b)fluoranthene (MW $252 \mathrm{u}$ ). Benzo(a)pyrene (MW $252 \mathrm{u}$ ) was present in the soil at $410 \mu \mathrm{g} / \mathrm{g}$. The high-molecular-weight PAHs measured in Tables 3 and 4 were typically 1-2 orders of magnitude less abundant than the low-molecular-weight PAHs in this particular sample.

\section{Comparison of Two PAH-Contaminated Soils}

Figures 7,8 , and 9 display the SIM PB LC-MS ion chromatograms at $m / z 326,352$, and 376 , respectively,

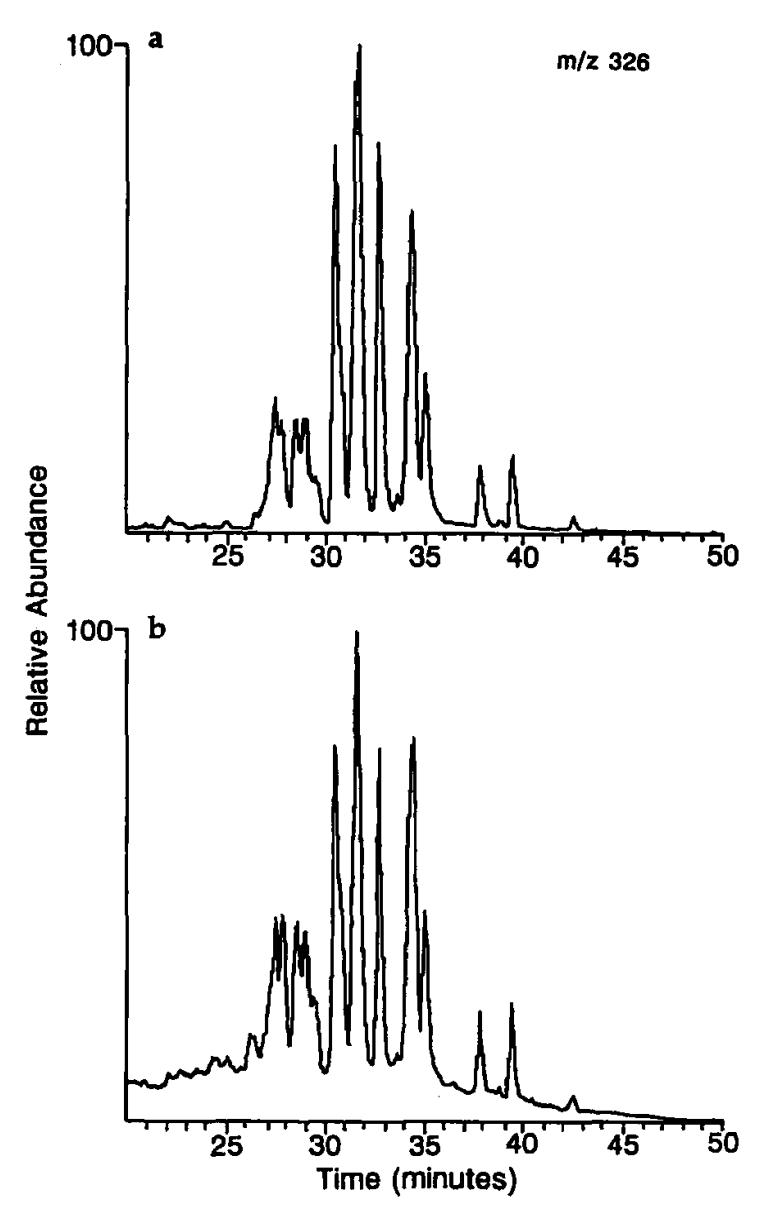

Figure 7. SIM ion chromatograms for $m / z 326$ of soil extracts from (a) the Pacific Northwest and (b) the northeast areas of the United States.

from extracts of two different environmental samples. Chromatogram a in each figure is from the Pacific Northwest soil described previously; chromatograms b are from a soil sample obtained at a hazardous waste site located in the northeastern United States. This second sample was prepared for analysis by the Contract Laboratory Program (CLP). The CLP methylene chloride extract was known to contain high levels of low-molecular-weight PAHs and was analyzed here to see if high-molecular-weight PAHs were present.

When the ion chromatograms from the two samples in each of Figures 7-9 are compared, some striking similarities are apparent. The chromatograms in these figures not only appear to contain the same isomers at each of the three molecular weights, but also the same relative areas. These similarities also appeared in the other ions monitored (not shown). One possible explanation for the similarities is that the two samples were collected from sites with similar types of contaminating materials. If this is the case, PB LC-MS could possibly be used to identify sources of contamination through pattern recognition. Another possible explanation is that in the formation of high-molecular-weight PAHs a selective process dictates what isomers are formed and their relative abundances. Wise et al. [15] 


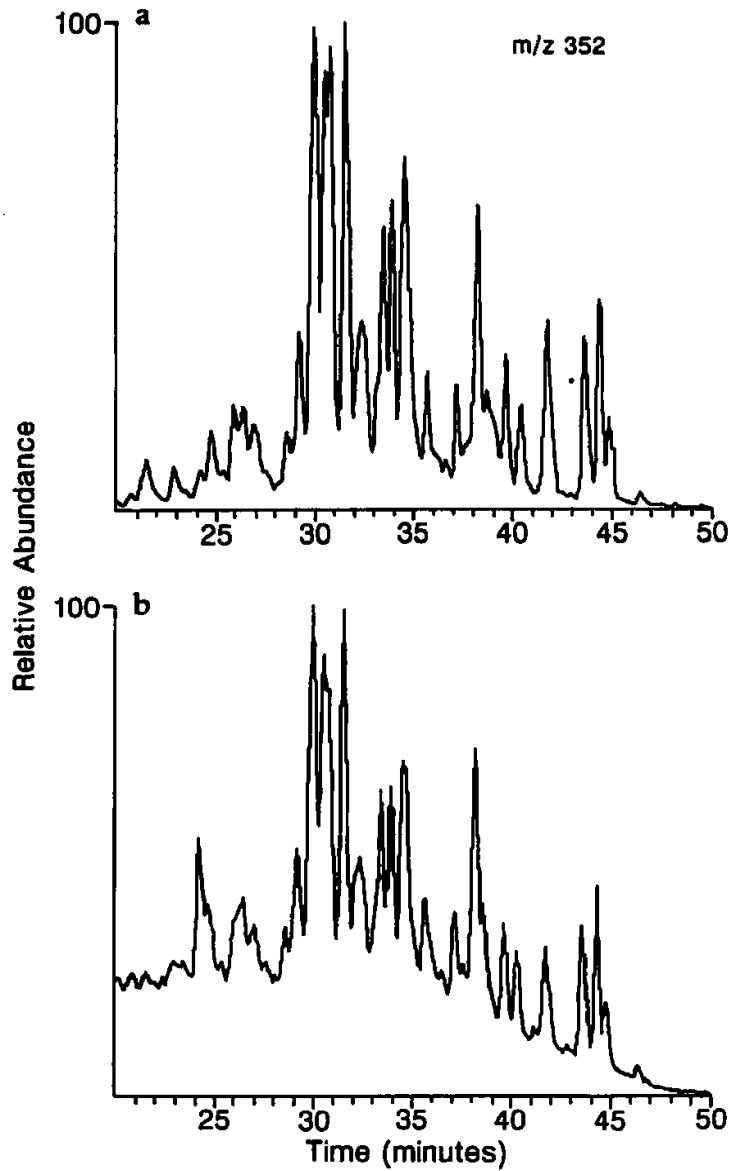

Figure 8. SIM ion chromatograms for $m / z 352$ of soil extracts from (a) the Pacific Northwest and (b) the northeast areas of the United States.

showed that similar relative concentrations for nine PAHs with molecular weight $302 \mathrm{u}$ existed in four environmental standard reference materials.

\section{Conclusion}

The results of this study show that PB LC-MS can be a valuable tool for the monitoring of high-molecularweight PAHs. PB LC-MS exhibited good sensitivity to the PAHs. This sensitivity is sufficient to measure high-molecular-weight PAHs at levels present in many environmental samples. It is clear in this work and from the literature that conventional HPLC columns do not have the efficiency necessary to separate the bulk of the high-molecular-weight PAH components likely to be present in a complex real-world sample. The solution may lie in increased selectivity through combinations of multidimensional chromatographic approaches and selective detection (mass spectrometry, UV, fluorescence). The PAH compounds used in these studies, although suitable for characterization of instrument performance and sample preparation schemes, were selected primarily on the basis of the availability of pure standards, not on the basis of environmental relevance. The selection of a limited set

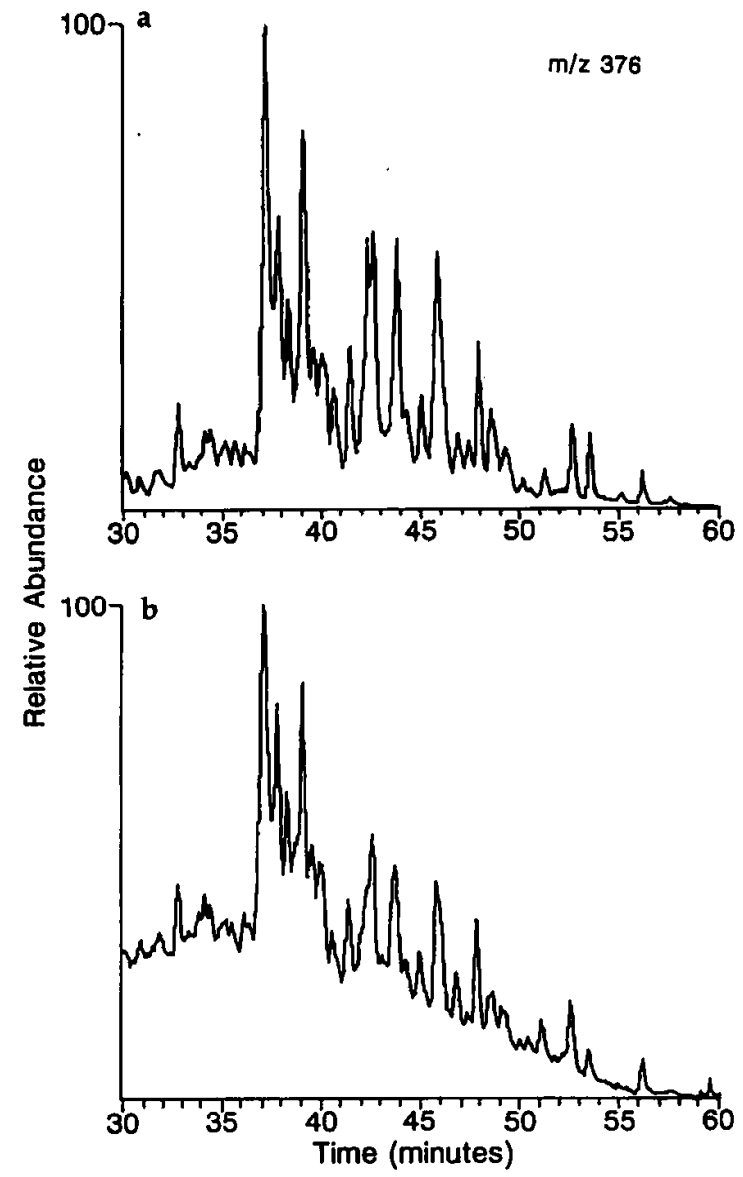

Figure 9. SIM ion chromatograms for $m / z 376$ of soil extracts from (a) the Pacific Northwest and (b) the northeast areas of the United States.

of environmentally relevant, high-molecular-weight PAHs should be based on toxicity, frequency of occurrence in the environment, and the extent of occurrence.

\section{References}

1. Harvey, R. G.; Polycyclic Hydrocarbons and Carcinogenesis, ACS Symposium Series 283; American Chemical Society: Washington, D.C., 1985.

2. Leger, A.; d'Hendecourt, L. Astron. Astrophys. 1985, 146, 81-85.

3. Clar, E. Polycyclic Hydrocarbons, Academic Press: London, 1964.

4. Lacassange, A.; Buu-Hoi, N. P.; Zadjela, F.; Saint-Ruf, G. Acad. Sci. Paris Ser. D 1968, 266, 301.

5. Grimmer, G.; Brune, H.; Deutsch-Wentzel, R.; Dettbarn, G.; Misfeld, J.; Abel, U.; Timm, J. Cancer Lett. 1984, 23, 167.

6. Schmidt, W.; Grimmer, G.; Jacob, J.; Dettbarn, G.; Naujack, K. W. Fresenius Z. Anal. Chem. 1987, 326, 401-413.

7. Peaden, P. A.; Lee, M. L.; Hirata, Y.; Novotny, M. Anal. Chem. 1980, 52, 2268-2271.

8. Hirata, Y.; Novotny, M.; Peaden, P. A.; Lee, M. L. Anal. Chim. Acta 1981, 127, 55-61.

9. Hirose, A.; Wiesler, D.; Novotny, M. Chromatographia 1984, $18,239-242$.

10. Colmsjo, A. L.; Ostman, C. E. Anal. Chim. Acta 1988, 208, 183-193. 
11. Wise, S. A.; Benner, B. A.; Liu, H.; Byrd, G. D.; Colmsjo, A. L. Anal. Chem. 1988, 60, 630-637.

12. Romanowski, T.; Funcke, W.; Grossman, I.; Konig, J.; Balfanz, E. Anal. Chem. 1983, 55, 1030-1033.

13. Fetzer, J. C.; Biggs, W. R.; Jinno, K. Chromatographia 1986, 21, 439-442.

14. Wright, B. W.; Udseth, H. R.; Chess, E. K.; Smith, R. D. J. Chromatogr. Sci. 1988, 26, 228-235.

15. Wise, S. A.; Deissler, A.; Sander, L. C. I. Polycyclic Aromatic Compounds 1993, 3, 169-184.

16. Doerge, D. R.; Clayton, J.; Fu, P. P.; Wolfe, D. A. Biol. Mass Spectrom. 1993, 22, 654-660.

17. Perreault, H.; Ramaley, L.; Sim, P. G.; Benoit, F. M. Rapid Commun. Mass Spectrom. 1991, 5, 604-610.

18. Pace, C. M.; Miller, D. A.; Roby, M. R. Measurement of Polycyclic Aromatic Hydrocarbons in Soils and Sediments by
Particle-Beam/High-Performance Liquid Chromatography/Mass Spectrometry; EPA 600/4-92/032; U.S. Environumental Protection Agency; Las Vegas, NV, 1992.

19. Bellar, T. A.; Behymer, T. D.; Budde, W. L. J. Am. Soc. Mass Spectrom. 1990, 1, 92-98.

20. Wise, S. A.; Bonnett, W. J.; Guenther, F. R.; May, W. E. J. Chromatogr. Sci. 1981, 19, 457-465.

21. Jinno, K.; Nagoshi, T.; Tanaka, N.; Okamoto, M.; Fetzer, J. C.; Biggs, W. R. J. Chromatogr. 1987, 386, 123-135.

22. Jinno, K.; Nagoshi, T.; Tanaka, N.; Okamoto, M.; Fetzer, J. C.; Biggs, W. R. J. Chromatogr. 1987, 402, 173-178.

23. Fetzer, J. C.; Biggs, W. R. J. Chromatogr. 1984, 295, 161-169.

24. Fetzer, J. C.; Biggs, W. R. Chromatographia 1989, 27, 118-122.

25. Jinno, K.; Nagoshi, T.; Tanaka, N.; Okamoto, M.; Fetzer, J. C.; Biggs, W. R. J. Chromatogr. 1988, 436, 1-10.

26. Cappiello, A.; Bruner, F. Anal. Chem. 1993, 65, 1281-1287. 